\title{
An integrated intervention for chronic care management in rural Nepal: protocol of a type 2 hybrid effectiveness-implementation study
}

Dan Schwarz ${ }^{1,2,3,4,5^{*+}} \mathbb{D}^{\text {D }}$ Santosh Dhungana ${ }^{6+}$, Anirudh Kumar ${ }^{7}$, Bibhav Acharya ${ }^{1,8}$, Pawan Agrawal ${ }^{1}$, Anu Aryal ${ }^{1,9}$, Aaron Baum ${ }^{10}$, Nandini Choudhury ${ }^{1,10}$, David Citrin 1,10,11,12,13, Binod Dangal ${ }^{1}$, Meghnath Dhimal ${ }^{14}$, Bikash Gauchan ${ }^{1}$, Tula Gupta ${ }^{1,15}$, Scott Halliday 10,111, Biraj Karmacharya 9,16,17, Sandeep Kishore ${ }^{10,18,19}$, Bhagawan Koirala ${ }^{20}$, Uday Kshatriya $^{1}$, Erica Levine ${ }^{10,18}$, Sheela Maru 1,10,18,21,22, Pragya Rimal', Sabitri Sapkota ${ }^{1,10}$, Ryan Schwarz ${ }^{1,2,3,22}$, Archana Shrestha ${ }^{9,23,24}$, Aradhana Thapa ${ }^{1}$ and Duncan Maru1,10,1,8,25,26

\begin{abstract}
Background: In Nepal, the burden of noncommunicable, chronic diseases is rapidly rising, and disproportionately affecting low and middle-income countries. Integrated interventions are essential in strengthening primary care systems and addressing the burden of multiple comorbidities. A growing body of literature supports the involvement of frontline providers, namely mid-level practitioners and community health workers, in chronic care management. Important operational questions remain, however, around the digital, training, and supervisory structures to support the implementation of effective, affordable, and equitable chronic care management programs.

Methods: A 12-month, population-level, type 2 hybrid effectiveness-implementation study will be conducted in rural Nepal to evaluate an integrated noncommunicable disease care management intervention within Nepal's new municipal governance structure. The intervention will leverage the government's planned roll-out of the World Health Organization's Package of Essential Noncommunicable Disease Interventions (WHO-PEN) program in four municipalities in Nepal, with a study population of 80,000. The intervention will leverage both the WHO-PEN and its cardiovascular disease-specific technical guidelines (HEARTS), and will include three evidence-based components: noncommunicable disease care provision using mid-level practitioners and community health workers; digital clinical decision support tools to ensure delivery of evidence-based care; and training and digitally supported supervision of mid-level practitioners to provide motivational interviewing for modifiable risk factor optimization, with a focus on medication adherence, and tobacco and alcohol use. The study will evaluate effectiveness using a pre-post design with stepped implementation. The primary outcomes will be disease-specific, "at-goal" metrics of chronic care management; secondary outcomes will include alcohol and tobacco consumption levels.

(Continued on next page)
\end{abstract}

\footnotetext{
* Correspondence: daschwarz@gmail.com

†Dan Schwarz and Santosh Dhungana are co-first authors

${ }^{1}$ Nyaya Health Nepal, Kathmandu, Nepal

${ }^{2}$ Division of Global Health Equity, Department of Medicine, Brigham and

Women's Hospital, 75 Francis Street, Boston, MA, USA

Full list of author information is available at the end of the article
}

(c) The Author(s). 2020 Open Access This article is distributed under the terms of the Creative Commons Attribution 4.0 International License (http://creativecommons.org/licenses/by/4.0/), which permits unrestricted use, distribution, and reproduction in any medium, provided you give appropriate credit to the original author(s) and the source, provide a link to the Creative Commons license, and indicate if changes were made. The Creative Commons Public Domain Dedication waiver (http://creativecommons.org/publicdomain/zero/1.0/) applies to the data made available in this article, unless otherwise stated. 
(Continued from previous page)

Discussion: This is the first population-level, hybrid effectiveness-implementation study of an integrated chronic care management intervention in Nepal. As low and middle-income countries plan for the Sustainable Development Goals and universal health coverage, the results of this pragmatic study will offer insights into policy and programmatic design for noncommunicable disease care management in the future.

Trial registration: ClinicalTrials.gov, NCT04087369. Registered on 12 September 2019.

Keywords: Noncommunicable diseases, Chronic illness, Community health workers, Decision support systems, Motivational interviewing, Rural health, Nepal

\section{Background}

The burden of noncommunicable diseases (NCDs) is rising globally [1, 2], and four major NCD classes-cardiovascular disease, chronic respiratory disease, diabetes, and cancer-contribute to more deaths globally than all other diseases combined, with enormous health and economic implications currently and in the future [3, 4]. This is especially true in low and middle-income countries (LMICs), where governments are struggling to plan for the increasing health and financial implications of these pandemics [5]. As governments strive to achieve the Sustainable Development Goals [6] and work toward universal health coverage [7], the rising NCD pandemic poses significant barriers to these aspirations.

In spite of this growing need for long-term chronic disease care, LMIC healthcare systems are not well equipped to address this disease burden [8]. Historically focused on delivering acute, episodic care, these healthcare systems lack the capacity to provide ongoing longitudinal care for patients with diseases best managed across a lifetime. This is especially true for patients with multiple chronic conditions, who often struggle to receive care for comorbid diseases without numerous, fragmented encounters [9]. Health workforce shortages and an emphasis on physician-provided care have contributed to significant access, coverage, and quality deficits $[10,11]$.

There is a growing body of evidence that chronic diseases in LMICs can be well managed, especially at the primary care level, by mid-level practitioners (MLPs) [10, 12-21]. MLPs offer a feasible, affordable, and highquality alternative to traditionally physician-delivered care. This can help to circumvent access barriers, thereby leading to fewer late-stage presentations and complications of otherwise manageable chronic conditions [20]. Evidence suggests that the quality of MLPdelivered care improved when they are well-managed, assigned clear scopes of work for which they are appropriately trained, and receive supportive supervision [2224] from senior clinicians with continual, improvementfocused feedback [20].

Simultaneously, the role of community health workers (CHW) is increasingly recognized as an important augmentation to facility-based care delivery strategies, offering critical linkages, referrals, and coordination of care within communities [25-27]. The potential value of $\mathrm{CHW}$-augmented chronic care management has only recently become of major interest [12-18, 27-29]. Global experience suggests that the greatest benefits come from CHWs when they are accredited, well-managed, salaried, continually trained and supervised, integrated into strong primary care systems, and part of continual data feedback loops with facility-based providers [25].

Taken together, the need and opportunity for LMIC healthcare systems-already struggling with workforce shortages-to build strong chronic care programs managed by MLPs and augmented with CHWs are compelling. However, there is a paucity of large-scale implementation data available to evaluate such models of care delivery, making it difficult for LMIC policymakers to decide whether to adopt these strategies.

In response to the growing need for evidence-based NCD service delivery, the World Health Organization has compiled a set of protocols within the Package of Essential Noncommunicable Disease Interventions (WHOPEN) for primary health care in low-resource settings $[30,31]$. These protocols constitute simple decisionmaking algorithms for the screening, diagnosis, and management of major NCDs, helping to ensure quality care provision in primary care settings, especially in areas already struggling with workforce shortages.

The WHO, together with the World Heart Federation, the World Stroke Organization, the United States Centers for Disease Control, the International Society of Hypertension, and the World Hypertension League, has released complementary guidelines to the WHO-PEN, called HEARTS [32]. (The acronym stands for: Healthy-lifestyle counselling. Evidence-based treatment protocols. Access to essential medicines and technology. Risk-based charts. Team-based care. Systems for monitoring.) In addition to bringing specific guidelines for cardiovascular disease management into the technical base of WHO-PEN, these guidelines provide expanded details around health information systems, workforce development and training, and service delivery. As such, they augment the systems focus of WHO-PEN in incorporating NCD care into broader healthcare systems strengthening. 
Recent evidence has shown the feasibility of adopting WHO-PEN at the primary care level in LMICs, including implementation with MLPs [33-36]. These data suggest that it is feasible to deploy WHO-PEN at the population level in primary care settings. While WHOPEN and HEARTS do not focus explicitly on the engagement of $\mathrm{CHWs}$ in these care delivery chains, there is a strong potential for augmenting MLP-based WHOPEN and HEARTS care at facilities with CHW-based strategies in the community.

Clinical decision support (CDS) tools facilitate the use of algorithmic care protocols such as WHO-PEN by health workers at the point of care. HEARTS provides specific CDS algorithms for cardiovascular conditions. These algorithmic CDS tools are used globally, across high-income and LMIC settings, in a range of conditions, including heart disease and diabetes [37-42]. CDS tools are essential both for managing individual conditions as well as for structuring the comprehensive care of patients with multiple comorbidities. In addition to CDS tools integrated into electronic health records (EHRs), there is a growing body of evidence to support mobile-phone-based CDS tools, often targeted at MLPs [43] and CHWs [44]. These CDS tools are especially well equipped for clinical situations in which clear algorithms exist, such as the WHO-PEN and HEARTS protocols.

Historically, in many LMICs, there has been scant emphasis placed on risk factor reduction through lifestyle modification for NCD prevention or to reduce risk factors for the progression of these diseases when already diagnosed. While many of the contributing etiologies for NCD epidemics are far upstream of individuals' lives, and out of their control [45], there are certain risk factors that are modifiable by patients, families, and health workers. Among others, it is widely documented that alcohol and tobacco consumption directly contribute to the development and progression of cardiovascular disease, diabetes, and chronic obstructive pulmonary disease (COPD) [46]. These risk factors have been marked as priority targets for NCD control moving forward, including in both the WHO-PEN and HEARTS protocols $[8,47]$.

WHO-PEN Protocol \#2 (republished in HEARTS), "Health Education and Counseling on Healthy Behaviors," describes risk factor modification [31]. However, while emphasizing health education, there is often very little guidance provided to health workers for meaningful engagement with patients and communities to accomplish these difficult lifestyle modification goals. There are many limitations to purely knowledge-focused approach health education, versus one that emphasizes self-efficacy and patients' own values [48].

Motivational interviewing (MI), originally validated in substance-abuse interventions, approaches behavior change through a style characterized by empathy and collaboration aimed toward the patient's readiness for change [49]. Trial data demonstrate the efficacy of MI for behavior change such as smoking and alcohol cessation [50-55]. These data raise the possibility of MI program development, led by MLPs within LMICs, as a viable strategy for improving risk factor modification interventions, and, specifically, as an augmentation to WHO-PEN, to strengthen its impact. Since many communities struggle with a lack of healthy nutritional options, dietary improvements are often difficult to realize. Reducing alcohol and tobacco consumption, however, are realistic options for risk factor modification interventions in even the poorest communities worldwide. Recent WHO-PEN guidance on "brief interventions" for alcohol and tobacco, in concert with MI-related interventions, offer practical options for addressing these challenges in the primary care setting [48].

In Nepal, the burden of NCDs is rapidly growing [5658], within the context of an overburdened healthcare system $[12,58,59]$. Nepal's government is committed to achieving the Sustainable Development Goals and universal healthcare, and has a specific focus on expanding health services for NCDs. In addition to recently enshrining the right to healthcare into its Constitution [60], Nepal's government has committed to addressing the NCD epidemic by launching a Multisectoral Action Plan in 2014 [61] and establishing the Nepal Noncommunicable Disease \& Injury Poverty Commission in 2016 [58]. Furthermore, the Ministry of Health and Population has committed to a step-wise national implementation of PEN $[58,59,62]$. Nonetheless, the way forward will be challenging, and innovative strategies are clearly needed to accomplish these ambitious goals [58].

Nepal has an extensive history of both MLP and CHW interventions [63-65]. Recent evidence from within the country has demonstrated the potential for CHWs to be involved in hypertension management [66]. Many communities rely on MLPs for primary care $[43,67]$, and there has been some experience with MLPs using CDS tools for algorithmic care provision [43]. However, to date there are no large-scale implementation data for integrated, MLP-based and CHWbased NCD care management at the population level. Similarly, while there has been some anecdotal description of MI being utilized in urban areas for select populations, there is no population-level data surrounding the use of MI for NCD risk modification. As the country endeavors to develop cross-sectoral strategies to address the growing NCD epidemic, these staffing models, coupled with CDS tools and MI-based adherence and risk modification, are important policy and programmatic considerations. 
We will conduct a type 2 hybrid effectivenessimplementation trial (where effectiveness and implementation are simultaneously tested with equal priority simultaneously) $[68,69]$ to evaluate an integrated NCD care management intervention. The intervention will leverage the Nepali government's planned roll-out of WHO-PEN in two rural districts. In addition to the government's roll-out, the intervention will include three evidencebased components: NCD care provision by MLPs and CHWs that is integrated between facilities and communities; CDS tools for MLPs and CHWs to optimize adherence to best practices; and training and supervision of MLPs in using MI to facilitate tobacco and alcohol cessation.

\section{Methods}

\section{Study aims}

As already stated, we will conduct a type 2 hybrid effectiveness-implementation trial to evaluate an integrated NCD care management intervention in rural Nepal. The intervention is described in depth in Additional file 1.

\section{Study implementers}

Healthcare workers and research staff from the nonprofit organization Nyaya Health Nepal, their collaborators in the Ministry of Health and Population and Nepal Health Research Council, and collaborating researchers form the study team will lead the study. Nyaya Health Nepal has been working in a public-private partnership with the Ministry of Health and Population for over 10 years in rural Nepal to deliver community-based and facility-based health services, and this study will leverage this pre-existing partnership and care delivery network. Nyaya Health Nepal operates with a US-based nonprofit organization, Possible, to advance national and global healthcare systems policy and practice priorities.

\section{Study setting}

The study will take place in the Achham and Dolakha districts of Nepal across four municipalities. Following recent healthcare decentralization, Nepal's 750 municipalities manage primary healthcare delivery. The intervention will be implemented in a step-wise fashion in coordination with municipal-level government authorities and study staff.

Achham is a remote, impoverished district of 260,000 people, with large migrant populations and a history of social disruption during the Nepali civil conflict [70-74]. Achham has one of the highest district-level under-5 mortality rates [75] and one of the lowest human development indices in the country [76]. The study implementers have been delivering some NCD-related care at the district-level Bayalpata Hospital and to communities within the hospital's catchment population since 2008 . Bayalpata Hospital serves approximately 90,000 outpatient and 3000 inpatient visits per year. CHW services include proactive case detection, care coordination, and counseling. The study will include a catchment population of approximately 50,000 in Achham across two municipalities.

The second district is Dolakha, one of the hardest hit districts in the 2015 earthquakes [77]. Nyaya Health Nepal's work in Dolakha is based at Charikot Primary Health Care Center, which serves approximately 60,000 outpatients per year, with similar $\mathrm{CHW}$ services to those in Achham's. The study will include a population of approximately 30,000 in Dolakha across two municipalities. Thus, the total expected study population will be 80,000 .

Within the context of the public-private partnership between the government and Nyaya Health Nepal, no user fees are charged for any facility-based or community-based services, in either Achham or Dolakha, thereby mitigating financial access barriers to care delivery and study participation.

Within the study setting, MLPs for the NCD intervention are locally defined as the Nepali cadre of health assistants, who have 3 years of postsecondary medical training. The CHWs in this intervention have secondary-school-level education and are fully employed, with ongoing supervision from community health nurses (CHNs). They receive initial training of approximately 1 month when they are hired, and ongoing weekly trainings to continually improve their skillsets. The CHWs are employed by the public-private partnership between Nyaya Health Nepal and the Ministry of Health and Population. They are distinct from the robust Female Community Health Volunteer network that exists throughout Nepal [63, 78], who have historically focused on vaccination, public health messaging, and other community preventive interventions rather than on household care delivery and follow-up. These staffing, supervision, and training structures are described in greater detail in Additional files 1 and 2.

\section{Study populations}

For primary quantitative outcomes, the study population will include adult patients ( $\geq 18$ years of age) who qualify for a diagnosis of hypertension, type II diabetes, and/or COPD, according to WHO-PEN guidelines, and are engaged in longitudinal care by Nyaya Health Nepal's team in Achham and Dolakha. The study will limit enrollment to the catchment areas served by both the facility-level and CHW-level services deployed by Nyaya Health Nepal. Study participants will be initially enrolled during facility-based visits at Bayalpata Hospital and Charikot Primary Health Care Center prior to the completion of intervention roll-out, and are considered engaged in 
longitudinal care if they have at least one follow-up hospital visit after 12 months of their initial visit. Digital health records that link between the facility-based EHR and the CHWs' mobile-phone applications will be utilized to share patient data across settings, when available. CHWs can identify potential patients in the community and refer them to the facility for diagnosis confirmation, following which they could be included in the study. Patients' receipt of care will not be contingent upon their enrollment in the study; all patients will continue to receive care per routine service delivery. This represents an exhaustive convenience sampling method as all eligible patients identified at Bayalpata Hospital and Charikot Primary Health Care Center may be enrolled in the study. Exclusion criteria are individuals planning to migrate from the study area prior to 12 months of exposure to the intervention, or individuals explicitly requesting exclusion from the study or declining to consent (see Additional file 3) for the study.

For implementation components, staff members, patients, community leaders, and government officials will be approached for key-informant interviews (KIIs) and focus group discussions (FGDs), as described in the following.

\section{Study design}

This is a prospective, mixed-methods type 2 hybrid effectiveness-implementation study to evaluate an integrated NCD care management intervention. We plan to apply both qualitative and quantitative methods in a complementary manner [79], in order to meaningfully assess both patient-level and population-level outcomes and the effectiveness of the implementation strategy. We will study the intervention's impact on patients' disease management outcomes after 12 months of being enrolled in NCD care using a pre-post design across both sites:

1. We will study the implementation of the intervention utilizing both quantitative and qualitative methods applying the RE-AIM (Reach, Effectiveness, Adoption, Implementation, Maintenance) framework [80].

Data collection is developed and integrated within the routine course of delivering care, which is an ethical, acceptable, and affordable approach in this setting. See Fig. 1 for a trial flowchart and Additional file 4 for a SPIRIT research reporting checklist. It is not feasible nor ethically acceptable to obtain data on a comparison (control) group in this population. Given the lack of national or local NCD systems, no data are available from other sources prior to the start of the study.

\section{Study outcomes}

The study has two specific aims: effectiveness (Specific Aim 1) and implementation (Specific Aim 2), as detailed in Table 1. For Specific Aim 1, the primary outcome will be the proportion of patients who meet disease-specific, evidence-based control measures at the completion of their initial 12 months engaged in treatment. These "atgoal" metrics aim to serve as simplified measures to assess disease control status, recognizing the limitations associated with multiple disease-specific metrics in settings like rural Nepal, especially for patients with multiple comorbid conditions. These are presented in Table 2.

Secondary outcomes for Specific Aim 1 will include the following. We will assess the individual "at-goal" rates per condition. We will assess the persistence of the intervention for the subset of patients for whom we have the data (i.e., those enrolled within 12 months of the start of the study) on their 24 months' outcome. Additionally, we will examine the tobacco and alcohol status of enrolled patients, specifically focusing on the proportion of patients who were tobacco users and/or alcohol drinkers at the time of enrollment who have stopped all tobacco and/or alcohol intake, or reduced their intake by $>50 \%$, by the completion of the study period (Table 1).

For Specific Aim 2, the RE-AIM framework will be utilized to assess the implementation of the study intervention, with RE-AIM metrics as presented in Table 1.

\section{Sampling strategy and power calculations}

We will use exhaustive convenience sampling to screen all eligible patients seen across two facilities over a 12-month period into the analysis cohort. Based on historical formative data of patient volume seen at these two facilities, and accounting for an expected $30 \%$ attrition rate, we conservatively expect that at least 1000 patients will be eligible for enrollment into the cohort.

With this conservative number of 1000 as our expected sample size based on this convenience sampling, we conducted power calculations to determine the statistical power to detect a change in the "at-goal" status. We calculate power based on a simplified design to compare paired proportions using a two-sided McNemar's test with an 0.05 type I error $(\alpha)$ level. The primary outcome is the proportion of patients who achieve their NCD control target ("at-goal status") after 12 months of being engaged in care. We used SAS version 9.4 (Cary, NC, USA) to estimate power to detect a $5 \%$ difference between discordant proportions; that is, proportions of patients whose "at-goal" status changed from "not at-goal" at baseline to "at-goal" at follow-up, and vice versa, in multiple scenarios where the total proportion of discordant patients ranged from 10 to $40 \%$ of all 


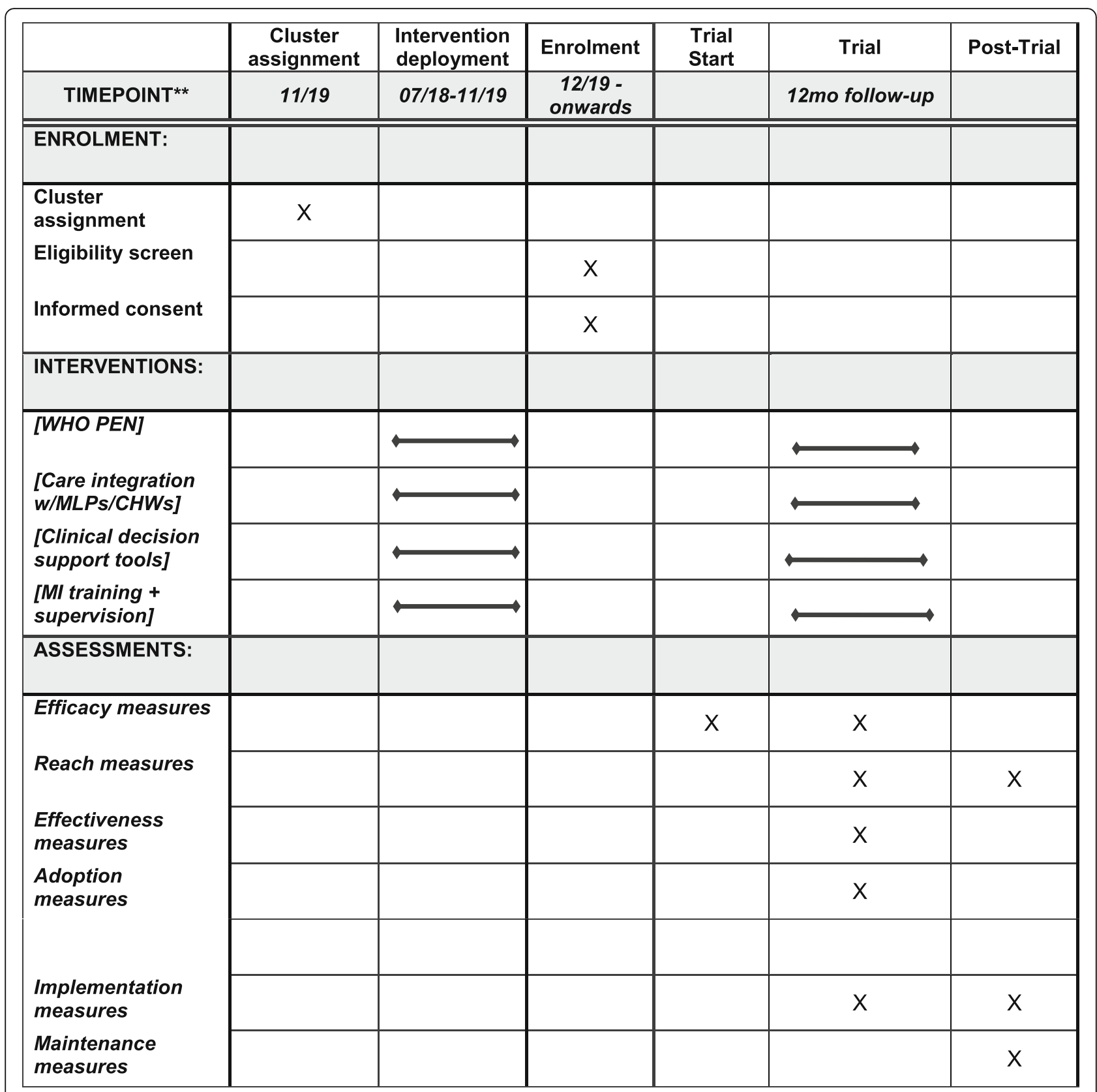

Fig. 1 Standard Protocol Items: Recommendations for Interventional Trials (SPIRIT) figure. [81] Recommended content can be displayed using various schematic formats. See SPIRIT 2013 Explanation and Elaboration for examples from protocols. **List specific timepoints in this row

patients. Based on these assumptions, our power to detect a $5 \%$ difference in the discordant pairs is $69 \%$, when the total discordant proportion is $40 \%$, and the power is $99 \%$ when $10 \%$ of all patients are discordant.

\section{Data collection}

\section{Quantitative data}

Quantitative data for patient outcomes will be extracted from the facility-based EHR and the CHW's mobile-phone application (Additional file 5), and will be used to assess
Specific Aims 1 and 2. (Table 1) All implementationrelated data for evaluating the performance of MLPs and CHWs (Table 1) will be collected by the responsible MLP and $\mathrm{CHW}$ supervisors in digitized checklists within the EHR and mobile-phone application. Access to protected health information will be controlled and defined by user access groups according to clinician status. Data to be analyzed will be extracted via secure data queries from the EHR system in an aggregate, partially de-identified form, with external researchers signing a data-sharing and use 
Table 1 Metrics for Specific Aim 1 (efficacy) and Specific Aim 2 (implementation)

\begin{tabular}{|c|c|c|c|}
\hline Aim & $\begin{array}{l}\text { Outcome/RE-AIM } \\
\text { element }\end{array}$ & Indicator & Definition \\
\hline \multirow[t]{3}{*}{$\begin{array}{l}\text { Specific Aim 1: } \\
\text { efficacy }\end{array}$} & $\begin{array}{l}\text { Primary outcome: } \\
\text { control of NCD } \\
\text { conditions }\end{array}$ & $\begin{array}{l}\text { Condition-specific "at-goal" } \\
\text { metrics }\end{array}$ & $\begin{array}{l}\text { \% of enrolled NCD patients achieving "at-goal" status (Table 2), } \\
\text { at completion of the study period }\end{array}$ \\
\hline & $\begin{array}{l}\text { Secondary outcome 1: } \\
\text { tobacco use }\end{array}$ & Tobacco use status & $\begin{array}{l}\% \text { of enrolled NCD patients who were using tobacco at enrollment } \\
\text { who are nonusers or who have reduced by }>50 \% \text { their tobacco } \\
\text { intake, at completion of the study period }\end{array}$ \\
\hline & $\begin{array}{l}\text { Secondary outcome } 2 \text { : } \\
\text { alcohol use }\end{array}$ & Alcohol use status & $\begin{array}{l}\% \text { of enrolled NCD patients who were alcohol drinkers at enrollment } \\
\text { who are nondrinkers or who have reduced by }>50 \% \text { alcohol intake, } \\
\text { at completion of the study period }\end{array}$ \\
\hline \multirow[t]{16}{*}{$\begin{array}{l}\text { Specific Aim 2: } \\
\text { implementation }\end{array}$} & \multirow[t]{5}{*}{ Reach } & Home visit coverage & $\begin{array}{l}\% \text { of enrolled NCD patients having a CHW home visit, measured } \\
\text { monthly }\end{array}$ \\
\hline & & Clinic visit coverage & $\begin{array}{l}\% \text { of enrolled NCD patients having an MLP visit at the clinic, } \\
\text { measured monthly according to the patients indicated to be seen } \\
\text { that month based on protocol-based guidelines }\end{array}$ \\
\hline & & $\begin{array}{l}\text { Demographic, geographic } \\
\text { barriers and facilitators }\end{array}$ & $\begin{array}{l}\text { \% of enrolled NCD patients whose CHW has GPS-mapped their } \\
\text { households, describing barriers/facilitators to individuals' access, and } \\
\text { identifying contributors to variation/inequities }\end{array}$ \\
\hline & & Loss to follow-up & $\begin{array}{l}\% \text { of patients, stratified by demographic data and NCD conditions, } \\
\text { who are lost to follow-up after enrollment }\end{array}$ \\
\hline & & $\begin{array}{l}\text { Monthly patient touch- } \\
\text { points }\end{array}$ & $\begin{array}{l}\text { Number of monthly per-patient touch-points, including interactions } \\
\text { by both MLPs and CHWs }\end{array}$ \\
\hline & \multirow[t]{3}{*}{ Efficacy } & $\begin{array}{l}\text { Evidence-based hypertension } \\
\text { management }\end{array}$ & $\begin{array}{l}\% \text { of enrolled hypertension patients in accordance with } \\
\text { evidence-based recommendations, as prescribed by clinical } \\
\text { algorithms, assessed quarterly by EHR audits }\end{array}$ \\
\hline & & $\begin{array}{l}\text { Evidence-based diabetes } \\
\text { management }\end{array}$ & $\begin{array}{l}\% \text { of enrolled diabetes patients in accordance with evidence-based } \\
\text { recommendations, as prescribed by clinical algorithms, assessed } \\
\text { quarterly by EHR audits }\end{array}$ \\
\hline & & $\begin{array}{l}\text { Evidence-based COPD } \\
\text { management }\end{array}$ & $\begin{array}{l}\% \text { of enrolled COPD patients in accordance with evidence-based } \\
\text { recommendations, as prescribed by clinical algorithms, assessed } \\
\text { quarterly by EHR audits }\end{array}$ \\
\hline & \multirow[t]{4}{*}{ Adoption } & Village-cluster adoption & $\%$ of intended village clusters receiving intervention \\
\hline & & Timely adoption & $\begin{array}{l}\% \text { of intended village clusters rolling-out intervention within } 3 \text { months } \\
\text { of schedule, according to local governance decisions to } \\
\text { roll-out the intervention }\end{array}$ \\
\hline & & CHW adoption & $\begin{array}{l}\% \text { of } \mathrm{CHWs} \text { trained in intervention implementation within the first } \\
6 \text { months } \\
\% \text { of trained CHWs retained in their positions at completion of the } \\
\text { study period }\end{array}$ \\
\hline & & MLP adoption & $\begin{array}{l}\% \text { of MLPs trained in intervention implementation } \\
\% \text { of trained MLPs retained in their positions at completion of the study } \\
\text { period }\end{array}$ \\
\hline & \multirow[t]{4}{*}{ Implementation } & Care integration & $\begin{array}{l}\% \text { of all NCD patients enrolled at the facilities seen by CHWs at home } \\
\text { within the first month }\end{array}$ \\
\hline & & CHW supervision model & $\begin{array}{l}\% \text { of scheduled CHW supervision field visits completed, stratified by CHN } \\
\text { and district, measured quarterly } \\
\% \text { of scheduled quarterly data review meetings held with CHWs and CHNs, } \\
\text { measured quarterly }\end{array}$ \\
\hline & & CHW home visit fidelity & $\begin{array}{l}\% \text { of enrolled NCD patients with } 100 \% \text { of algorithm-indicated home visits } \\
\text { received } \\
\% \text { of topics included at each session as dictated by the condition-specific } \\
\text { algorithms, assessed during the CHW supervision field visits by CHNs, } \\
\text { measured quarterly }\end{array}$ \\
\hline & & Referrals & $\begin{array}{l}\text { \% of patients appropriately referred to MLP care as indicated by the clinical } \\
\text { algorithms, assessed during the CHW supervision field visits by CHNs, } \\
\text { measured quarterly } \\
\% \text { of patients referred by CHWs seen by MLPs within the prescribed time } \\
\text { window according to the clinical algorithms (e.g., } 24 \text { h, } 72 \text { h, } 1 \text { week), }\end{array}$ \\
\hline
\end{tabular}


Table 1 Metrics for Specific Aim 1 (efficacy) and Specific Aim 2 (implementation) (Continued)

\begin{tabular}{|c|c|c|c|}
\hline \multirow[t]{2}{*}{ Aim } & $\begin{array}{l}\text { Outcome/RE-AIM } \\
\text { element }\end{array}$ & Indicator & Definition \\
\hline & \multicolumn{3}{|r|}{ measured quarterly } \\
\hline & & MLP supervision model & $\begin{array}{l}\% \text { of enrolled NCD patients appropriately referred to see a physician by } \\
\text { MLPs as indicated by the clinical algorithms, assessed during monthly } \\
\text { physician supervision sessions, measured quarterly }\end{array}$ \\
\hline & & MLP visit fidelity & $\begin{array}{l}\% \text { of enrolled NCD patients with } 100 \% \text { of algorithm-indicated facility } \\
\text { visits received, assessed during monthly physician supervision sessions, } \\
\text { measured quarterly } \\
\% \text { of diagnostic, treatment, and counseling topics included at each } \\
\text { session as dictated by the condition-specific algorithms, assessed during } \\
\text { monthly physician supervision sessions, measured quarterly }\end{array}$ \\
\hline & & Implementation challenges & $\begin{array}{l}\text { Exploratory and hypothesis-generating as revealed through FGDs and Klls } \\
\text { with CHWs, CHNs, MLPs, physicians, patients, and other relevant community } \\
\text { stakeholders }\end{array}$ \\
\hline & \multirow[t]{8}{*}{ Maintenance } & Total intervention cost & $\begin{array}{l}\text { Cost of each intervention component and total costs using the Joint } \\
\text { Learning Network costing methodology }\end{array}$ \\
\hline & & Intervention initiation costs & $\begin{array}{l}\text { \% breakdown of initial (one-time) costs for intervention (training, } \\
\text { equipment, etc.) }\end{array}$ \\
\hline & & $\begin{array}{l}\text { Intervention maintenance } \\
\text { costs }\end{array}$ & $\begin{array}{l}\text { \% breakdown of maintenance (recurring) costs (ongoing training, } \\
\text { personnel, materials, and other) }\end{array}$ \\
\hline & & Facility vs. community costs & $\begin{array}{l}\% \text { of costs of healthcare divided between facility level and community } \\
\text { level }\end{array}$ \\
\hline & & Geographic cost variation & $\begin{array}{l}\text { Characterization of variance in costs between village clusters and districts } \\
\text { within the intervention catchment area }\end{array}$ \\
\hline & & Out-of-pocket patient costs & $\%$ costs of healthcare divided between facility level and community level \\
\hline & & $\begin{array}{l}\text { Integrated intervention cost- } \\
\text { effectiveness analysis }\end{array}$ & Pre/post-intervention marginal effectiveness for primary outcomes \\
\hline & & Cost per unit & $\begin{array}{l}\text { Intervention cost per enrolled patient } \\
\text { Intervention cost per capita } \\
\text { Projected cost to scale intervention nationally, based on known incidence } \\
\text { and prevalence of each condition }\end{array}$ \\
\hline
\end{tabular}

$\overline{C H N}$ community health nurse, $C H W$ community health worker, COPD chronic obstructive pulmonary disease, EHR electronic health record, FGD focus group discussion, GPS Global Positioning System, KIl key-informant interview, MLP mid-level practitioner, NCD Noncommunicable disease, RE-AIM Reach, Effectiveness, Adoption, Implementation, Maintenance

agreement prior to analysis. Cleaned, de-identified datasets will be made publicly available via a data repository.

Costing data for the intervention will be collected utilizing a "top-down" method, as described by the Joint Learning Network [85]. This method will document direct and indirect costs associated with the NCD care delivery intervention described here, and related administrative functions (including planning and administration; training; supervision and monitoring and evaluation; data management; and continuous surveillance) will be disaggregated.

Table 2 Clinical definitions of "at-goal" status for each intervention condition

\begin{tabular}{lll}
\hline Noncommunicable disease & Management metric & "At-goal" definition \\
\hline Type II diabetes mellitus & Hemoglobin A1c OR fasting blood sugar & Hemoglobin A1c $<7.5$ OR fasting blood sugar $<130 \mathrm{mg} / \mathrm{dl}^{\mathrm{a}}$ \\
Hypertension & Blood pressure & $\begin{array}{l}\text { Blood pressure }<130 / 80 \mathrm{mmHg} \text { or patient-tailored goal per risk } \\
\text { stratification }\end{array}$ \\
Chronic obstructive pulmonary disease & Exacerbation status & $\leq 1 / 3$ Anthonisen criteriac $^{c}$
\end{tabular}

${ }^{a}$ The 2018 American Diabetes Association guidelines [82] call for a goal A1c $<7 \%$ for most patients or A1c < 8\% in "patients with a history of severe hypoglycemia, limited life expectancy, advanced microvascular or macrovascular complications, extensive comorbid conditions, or long-standing diabetes in whom the goal is difficult to achieve despite diabetes self-management education, appropriate glucose monitoring, and effective doses of multiple glucose-lowering agents including insulin." For our intervention, we established $7.5 \%$ as our goal to pragmatically accommodate both populations

${ }^{\mathrm{b}}$ Based on the 2017 American College of Cardiology and American Heart Association guidelines [83], we established < 130/80 mmHg as a default goal, with patient-tailored goals for select patients ( $\geq 65$ years of age, multiple comorbidities, limited life expectancy, clinical judgment, patient preference)

'The 2017 update to the GOLD guidelines [84] define chronic obstructive pulmonary disease exacerbation as an "acute worsening of respiratory symptoms that results in additional therapy." We used the Anthonisen criteria of worsening sputum volume, sputum purulence, and increased dyspnea to define the "worsening of respiratory symptoms" specified in the GOLD guidelines. We established a threshold of no more than one Anthonisen criterion as a pragmatic tool for determining clinical status 
Full methodology of direct and indirect costs is provided by the Joint Learning Network [85], and will be utilized for this study. For the purposes of this pragmatic study, this methodology will be appropriate to estimate the additional marginal costs of the intervention (rather than cost-savings or secondary cost implications) as compared to general standard of care.

\section{Qualitative data}

Qualitative data will be used for Specific Aim 2 (Table 1). Staff members, patients, community leaders, and government officials will be approached for KIIs and FGDs. Purposive sampling will be used, aiming to maximize heterogeneity across sex, socioeconomic position, healthcare issues, geographic location, age, caste class, and other attributes. For each group, five key informant interviews will be conducted at each time point, as described in the following. One focus group discussion per group will be conducted at each time point.

KII and FGD guides will be developed in advance, and will vary across the study period, exploring specific topics of concern. A locally validated, seven-domain framework of healthcare delivery analysis will be used to inform data collection [86]. These seven domains include health service operations, supply chains, equipment, personnel, outreach, societal factors, and structural factors. Qualitative data collection will focus on these areas to assess the implementation of the intervention.

FGDs and KIIs will occur prior to the initiation of the intervention, and at intervals of 6 months throughout the study period, to assess ongoing implementation status. All sessions will be conducted in Nepali. All qualitative data will be stored on a Research Electronic Data Capture (REDCap) database [87]. REDCap user access will be defined so that researchers only have access to de-identified study data. Any paper copies of data forms will be stored in locked cabinets inside locked rooms at district facilities. Once all data are fully transcribed and validated for quality, all paper copies will be destroyed. REDCap data will be deleted 12 months after the completion of the study period.

\section{Data analysis}

\section{Analysis for Specific Aim 1: effectiveness}

In order to assess the effectiveness of the intervention, as already described, the primary outcome will utilize disease-specific "at-goal" metrics for each of the three study diseases: hypertension, type II diabetes, and COPD. We hypothesize that the integrated intervention will lead to a $10 \%$ increase in the "at-goal" status of the combined disease cohorts, over a 12-month follow-up period.

We will use conditional multivariable logistic regression to assess patient outcomes at 12 months of followup, adjusting for potential confounding and/or effect modification by patients' demographics (including age, sex, caste), municipality, district, mean distance to the hospital, and engagement in care (defined as number of facilitybased and community-based encounters). We additionally hypothesize a $10 \%$ improvement in the status of each of the two secondary outcomes: tobacco and alcohol use, as measured by patient-reported outcomes presented in Table 1 .

As a secondary analysis for Specific Aim 1, namely the time-varying nature of the outcomes, we will assess the longitudinal effect of the intervention, as measured in 3monthly intervals, throughout the study period, compared to baseline statistics at the time of each villagecluster enrollment. Variables will be considered either nominal or continuous (linear effect) predictors, and the generalized linear model framework will be used to estimate effect of time-varying repeated-measure intervention implementation over the several steps of the wedged design. Differential impact from time of intervention will be evaluated with test of month $\times$ intervention interaction. Models will be fit using generalized estimating equations, for example, using SAS Proc Genmod, to calculate valid standard errors in the presence of repeated measures over time and possibly correlated outcomes at the municipality level. Assumptions of overdispersion or underdispersion will be examined closely, and an estimated scale parameter or negative binomial models will be used as needed.

\section{Analysis for Specific Aim 2: RE-AIM implementation framework}

In this mixed-methods study, Specific Aim 2 will be assessed using the RE-AIM framework for implementation trials [80]. A full list of metrics, separated by each domain of the REAIM framework, is presented in Table 1. Additional details regarding the supervision and audit structure for MLPs and CHWs can be found in Additional files 1 and 2.

For the maintenance of the intervention, we will assess the costs of the intervention, using the Joint Learning Network methodology [85]. Cost data will be analyzed and presented (Table 1) to help program planners and policy-makers understand the implications for possible scale of a similar intervention by the government or other entity in the future.

For quantitative data within Specific Aim 2, a similar methodology of generalized estimating equations, as already described in Analysis for Specific Aim 1: effectiveness, will be applied. Data will be assessed at 3-month intervals.

For qualitative data within Specific Aim 2, analysis will be ongoing and iterative, so as to continually inform further qualitative data collection, focusing on timely and relevant implementation issues. Data from KIIs and FGDs will transcribed and coded using grounded theory methodology $[88,89]$. NVivo software will be used for qualitative data analysis [90]. 


\section{Discussion}

\section{Ethical approval and consent}

This study has been approved by the Ethical Review Board of the Nepal Health Research Council (\#177/ 2018). Within the study, all patients will provide verbal informed consent to have their de-identified data analyzed and published. Care provision will be unrelated to consent, and there will be no difference in care provision based on consent status. Verbal informed consent will also be provided by all KII and FGD participants. No incentives will be provided to study participants, to avoid any conflict of interest or coercion to participate. Protocol modifications will be promptly communicated to the IRB and on the trial registry website by members of the research study team.

\section{Safety considerations}

There are minimal risks posed to patients, staff, or other key informants. The predominant risk is disclosure of protected health information, and/or qualitative data from KIIs or FGDs. All patient information will be stored on secure databases, and data access privileges will be heavily restricted. Unless otherwise deemed necessary for a specific analysis, all analyses will be conducted using a limited dataset. Qualitative data will be stored and protected as already described.

\section{Data sharing}

All de-identified data from this study will be made publicly available for other researchers to analyze at their discretion in the future, to further this field of research. De-identified summaries of qualitative data will be made available as well.

\section{Dissemination plan}

Domestically within Nepal, 6-monthly update meetings will be held between researchers and the Ministry of Health and Population to review ongoing results. When completed, results of the study will be presented at the annual National Summit of Health and Population Scientists, organized by the Nepal Health Research Council, and at other relevant international conferences. Peerreviewed publications will be drafted for international dissemination.

\section{Trial status}

At the time of manuscript submission, the study is currently not yet recruiting participants. Participant enrollment is anticipated to commence in February 2020 and is planned to continue for 1 year. Intervention deployment took place between July 2018 and will conclude in January 2020. This is study protocol version 1.2 and the version date is December 6, 2019.

\section{Supplementary information}

Supplementary information accompanies this paper at https://doi.org/10. 1186/s13063-020-4063-3.

Additional file 1. Detailed description of intervention.

Additional file 2. Community and facility-based supervision structures.

Additional file 3. Informed consent form.

Additional file 4. SPIRIT 2013 Checklist: Recommended items to address in a clinical trial protocol and related documents.

Additional file 5. Interview guides and data collection tools.

\section{Abbreviations}

CDS: Clinical decision support; CHN: Community health nurse;

CHW: Community health worker; COPD: Chronic obstructive pulmonary

disease; EHR: Electronic health record; FGD: Focus group discussion;

HEARTS: Healthy-lifestyle counselling. Evidence-based treatment protocols.

Access to essential medicines and technology. Risk based charts. Team-based care. Systems for monitoring; KII: Key-informant interview; LMIC: Low and middle-income country; MI: Motivational interviewing; MLP: Mid-level practitioner; NCD: Noncommunicable disease; RE-AIM: Reach, Effectiveness, Adoption, Implementation, Maintenance; REDCap: Research Electronic Data Capture; WHO: World Health Organization; WHO-PEN: WHO Package of Essential Noncommunicable Disease Interventions

\section{Acknowledgements}

The authors wish to express their appreciation to the Nepal Ministry of Health and Population for their continued efforts to improve the publicsector healthcare system in rural Nepal. They also wish to acknowledge the deep contributions of the patients, health workers, and community members, for whom all this work is designed and aims to serve.

\section{Authors' contributions}

Conceived the study: DS, SD, AK, NC, DC, SH, SK, RS, DM. Designed initial study protocol: DS, AK, NC, DC, SH, DM. Contributed to study protocol refinement: DS, SD, AK, BA, PA, AA, AB, NC, DC, BD, MD, BG, TG, SH, BKa, SK, BKo, UK, SM, PR, SS, RS, AS, AT, DM. Wrote the manuscript draft: DS, AK, NC, DM. Edited and revised the manuscript draft: DS, SD, AK, BA, PA, AA, AB, NC, DC, BD, MD, BG, TG, SH, BKa, SK, BKo, UK, EL, SM, PR, SS, RS, AS, AT, DM. Reviewed and approved the final manuscript draft: DS, SD, AK, BA, PA, AA, AB, NC, DC, BD, MD, BG, TG, SH, BKa, SK, BKo, UK, EL, SM, PR, SS, RS, AS, AT, DM.

\section{Funding}

This trial was conducted with no external funding and was instead funded by Nyaya Health Nepal with financial support from the Government of Nepal, Possible, and other private and philanthropic support. The authors received no direct funding in support of this manuscript.

\section{Availability of data and materials}

The datasets supporting the conclusions of the study will be made publicly available in de-identified form upon conclusion of the study. The final trial dataset (in limited identifier format) will be accessible to researchers at the research performance site in Nepal—Nyaya Health Nepal—and coinvestigators assisting with data analysis. Participating research institutions will enter into data-sharing agreements (namely between the research performance site in Nepal_Nyaya Health Nepal_and any foreign institutions where investigators are assisting with data analysis) covering terms of access to specific limited datasets; provisions for storing, sharing, and using data; and methods for securing data transfer.

\section{Ethics approval and consent to participate}

This study has been approved by the Ethical Review Board of the Nepal Health Research Council (\#177/2018). Within the study, all patients will provide verbal informed consent to have their de-identified data analyzed and published. Care provision will be unrelated to consent, and there will be no difference in care provision based on consent status. Verbal informed consent will also be provided by all KII and FGD participants. No incentives will be provided to study participants, to avoid any conflict of interest or coercion to participate. 


\section{Consent for publication}

Not applicable.

\section{Competing interests}

PA, AA, DC, BD, BG, TG, UK, PR, SS, and AT are employed by, and DS, BA, NC, $\mathrm{SH}, \mathrm{SM}, \mathrm{RS}$, and DM work in partnership with, a nonprofit healthcare company (Nyaya Health Nepal, with support from the US-based nonprofit organization, Possible) that delivers free healthcare in rural Nepal using funds from the Government of Nepal and other public, philanthropic, and private foundation sources. DS and RS are employed at an academic medical center (Brigham and Women's Hospital) that receives public-sector research funding, as well as revenue through private-sector fee-for-service medical transactions and private foundation grants. DS and RS are faculty members at a private medical school (Harvard Medical School). DS is employed at an academic medical center (Beth Israel Deaconess Medical Center) that receives public-sector research funding, as well as revenue through private-sector feefor-service medical transactions and private foundation grants. DS is employed at an academic research center (Ariadne Labs) that is jointly supported by an academic medical center (Brigham and Women's Hospital) and a private university (Harvard T.H. Chan School of Public Health) via publicsector research funding and private philanthropy. SD is a medical resident at a private academic medical center (Hurley Medical Center) that receives revenue through private-sector fee-for-service medical transactions and a charitable private foundation. AK is a medical resident at a private academic medical center (NYU Langone Health) that receives public-sector research funding, as well as revenue through private-sector fee-for-service medical transactions and private foundation grants. BA is a faculty member at a public university (University of California, San Francisco). AA is a fellow supported by a public-sector research fellowship affiliated at, and BKa and AS are faculty members at, a private university (Kathmandu University). DC is a faculty member at, $\mathrm{SH}$ is a graduate student at, and $\mathrm{DC}$ and $\mathrm{SH}$ are employed parttime at a public university (University of Washington). AB, DC, SK, SM, SS, and DM are faculty members at, and NC, SH, and EL are employed by, a private medical school (Icahn School of Medicine at Mount Sinai). MD is employed by the Government of Nepal (Ministry of Health and Population, Nepal Health Research Council). TG is a fellow with a bidirectional fellowship program (HEAL Initiative) that is affiliated with a public university (University of California, San Francisco) that receives funding from public, philanthropic, and private foundation sources. BKa is a faculty member at a public research university (Sun Yat-sen University). SK is the founding Executive Director at an advocacy and leadership network (Young Professionals Chronic Disease Network) that receives funding from individual philanthropy. SK serves as a consultant for Resolve To Save Lives on hypertension treatment and leads a partnership on multiple chronic conditions through his institution and Teva Pharmaceuticals. BKo is a faculty member at a public university (Tribhuvan University, Institute of Medicine). SM is a voting member on the Board of Directors with Group Care Global, a position for which she receives no compensation. RS is employed at an academic medical center (Massachusetts General Hospital) that receives public-sector research funding, as well as revenue through private-sector fee-for-service medical transactions and private foundation grants. AS is a faculty member at a private university (Yale School of Public Health). DM is a nonvoting member on Possible's Board of Directors, a position for which he receives no compensation. All authors have read and understood Trials' policy on declaration of interests, and declare that they have no competing financial interests. The authors do, however, believe strongly that healthcare is a public good, not a private commodity.

\section{Author details}

${ }^{1}$ Nyaya Health Nepal, Kathmandu, Nepal. ${ }^{2}$ Division of Global Health Equity, Department of Medicine, Brigham and Women's Hospital, 75 Francis Street, Boston, MA, USA. ${ }^{3}$ Department of Medicine, Harvard Medical School, Boston, MA, USA. ${ }^{4}$ Department of Medicine, Beth Israel Deaconess Medical Center, Boston, MA, USA. ${ }^{5}$ Ariadne Labs, Harvard T.H. Chan School of Public Health and Brigham and Women's Hospital, Boston, MA, USA. ${ }^{6}$ Department of Internal Medicine, Hurley Medical Center, Flint, MI, USA. ${ }^{7}$ Department of Medicine, NYU Langone Health, New York, NY, USA. ${ }^{8}$ Department of Psychiatry, University of California San Francisco, San Francisco, CA, USA. ${ }^{9}$ School of Medical Sciences, Kathmandu University, Dhulikhel, Nepal. ${ }^{10}$ Arnhold Institute for Global Health, Icahn School of Medicine at Mount Sinai, New York, NY, USA. ${ }^{11}$ Department of Global Health, University of Washington, Seattle, WA, USA. ${ }^{12}$ Department of Anthropology, University of
Washington, Seattle, WA, USA. ${ }^{13}$ Henry M. Jackson School of International Studies, University of Washington, Seattle, WA, USA. ${ }^{14}$ Nepal Health Research Council, Ministry of Health and Population, Kathmandu, Nepal. ${ }^{15}$ Health Equity Action Leadership Initiative, University of California, San Francisco, San Francisco, CA, USA. ${ }^{16}$ Nepal Technology Innovation Center, Kathmandu University, Dhulikhel, Nepal. ${ }^{17}$ Sun Yat-sen Global Health Institute, Sun Yat-sen University, Guangzhou, China. ${ }^{18}$ Department of Health Systems Design and Global Health, Icahn School of Medicine at Mount Sinai, New York, NY, USA. ${ }^{19}$ Young Professionals Chronic Disease Network, New York, NY, USA. ${ }^{20}$ Institute of Medicine, Tribhuvan University Teaching Hospital, Kathmandu, Nepal. ${ }^{21}$ Department of Obstetrics, Gynecology, and Reproductive Sciences, Icahn School of Medicine at Mount Sinai, New York, NY, USA. ${ }^{22}$ Division of General Internal Medicine, Department of Medicine, Massachusetts General Hospital, Boston, MA, USA. ${ }^{23}$ Yale School of Public Health, Center for Methods in Implementation and Prevention Science, New Haven, CT, USA. ${ }^{24}$ Department of Chronic Disease Epidemiology, Yale School of Public Health, New Haven, CT, USA. ${ }^{25}$ Department of Internal Medicine, Icahn School of Medicine at Mount Sinai, New York, NY, USA. ${ }^{26}$ Department of Pediatrics, Icahn School of Medicine at Mount Sinai, New York, NY, USA.

\section{Received: 28 September 2019 Accepted: 9 January 2020}

\section{Published online: 29 January 2020}

\section{References}

1. Kyu HH, Abate D, Abate KH, Abay SM, Abbafati C, Abbasi N, et al. Global, regional, and national disability-adjusted life-years (DALYs) for 359 diseases and injuries and healthy life expectancy (HALE) for 195 countries and territories, 1990-2017: a systematic analysis for the Global Burden of Disease Study 2017. Lancet. 2018;392(10159):1859-922.

2. Roth GA, Abate D, Abate KH, Abay SM, Abbafati C, Abbasi N, et al. Global, regional, and national age-sex-specific mortality for 282 causes of death in 195 countries and territories, 1980-2017: a systematic analysis for the Global Burden of Disease Study 2017. Lancet. 2018;392(10159):1736-88.

3. Global status report on noncommunicable diseases 2014. Geneva: World Health Organization; 2014.

4. Bloom DE, Cafiero ET, Jané-Llopis E, Abrahams-Gessel S, Bloom LR, Fathima $S$, et al. The global economic burden of noncommunicable diseases. Geneva: World Economic Forum and Harvard School of Public Health; 2011.

5. Muka T, Imo D, Jaspers L, Colpani V, Chaker L, van der Lee SJ, et al. The global impact of non-communicable diseases on healthcare spending and national income: a systematic review. Eur J Epidemiol. 2015;30(4):251-77.

6. United Nations. Sustainable Development Goals. https:// sustainabledevelopment.un.org/?menu=1300. Accessed 26 May 2016.

7. World Health Organization. Universal health coverage. https://www.who.int/ universal_health_coverage/en/. Accessed 24 Jan 2019.

8. Beaglehole R, Bonita R, Horton R, Adams C, Alleyne G, Asaria P, et al. Priority actions for the non-communicable disease crisis. Lancet. 2011;377(9775): 1438-47.

9. Hajat C, Kishore SP. The case for a global focus on multiple chronic conditions. BMJ Glob Health. 2018;3(3):e000874.

10. Lassi ZS, Cometto G, Huicho L, Bhutta ZA. Quality of care provided by midlevel health workers: systematic review and meta-analysis. Bull World Health Organ. 2013;91(11):824-833i.

11. Das J, Woskie L, Rajbhandari R, Abbasi K, Jha A. Rethinking assumptions about delivery of healthcare: implications for universal health coverage. BMJ. 2018;361:k1716.

12. Mishra SR, Neupane D, Preen D, Kallestrup P, Perry HB. Mitigation of noncommunicable diseases in developing countries with community health workers. Glob Health. 2015;11:43.

13. Abrahams-Gessel S, Denman CA, Gaziano TA, Levitt NS, Puoane T. Challenges facing successful scaling up of effective screening for cardiovascular disease by community health workers in Mexico and South Africa: policy implications. Health Syst Policy Res. 2016;3(1):26.

14. Tsolekile LP, Puoane T, Schneider H, Levitt NS, Steyn K. The roles of community health workers in management of non-communicable diseases in an urban township. Afr J Prim Health Care Fam Med. 2014;6(1):693.

15. Ndou T, van Zyl G, Hlahane S, Goudge J. A rapid assessment of a community health worker pilot programme to improve the management of hypertension and diabetes in Emfuleni sub-district of Gauteng Province, South Africa. Global Health Action. 2013;6:213-8. https://doi.org/10.3402/ gha.v3406i3400.19228. 
16. Joshi R, Alim M, Kengne AP, Jan S, Maulik PK, Peiris D, et al. Task shifting for non-communicable disease management in low and middle income countries - a systematic review. PLoS One. 2014;9(8):e103754.

17. Neupane D, Kallestrup P, CS ML, Perry H. Community health workers for non-communicable diseases. Lancet Global Health. 2015;2(10):e567.

18. Kane J, Landes M, Carroll C, Nolen A, Sodhi S. A systematic review of primary care models for non-communicable disease interventions in subSaharan Africa. BMC Fam Pract. 2017;18(1):46.

19. Abegunde DO, Mathers CD, Adam T, Ortegon M, Strong K. The burden and costs of chronic diseases in low-income and middle-income countries. Lancet. 2007;370(9603):1929-38.

20. Global Health Workforce Alliance. Mid-level health workers for delivery of essential health services: a global systematic review and country experiences. Geneva: World Health Organization; 2013.

21. Sharma KK, Gupta R, Mathur M, Natani V, Lodha S, Roy S, et al. Nonphysician health workers for improving adherence to medications and healthy lifestyle following acute coronary syndrome: 24-month follow-up study. Indian Heart J. 2016;68(6):832-40.

22. Manzi A, Magge H, Hedt-Gauthier BL, Michaelis AP, Cyamatare FR, Nyirazinyoye L, et al. Clinical mentorship to improve pediatric quality of care at the health centers in rural Rwanda: a qualitative study of perceptions and acceptability of health care workers. BMC Heath Serv Res. 2014;14(1):275.

23. Anatole M, Magge $H$, Redditt V, Karamaga A, Niyonzima S, Drobac P, et al. Nurse mentorship to improve the quality of health care delivery in rural Rwanda. Nurs Outlook. 2013:61(3):137-44.

24. Magge H, Anatole M, Cyamatare FR, Mezzacappa C, Nkikabahizi F, Niyonzima $S$, et al. Mentoring and quality improvement strengthen integrated management of childhood illness implementation in rural Rwanda. Arch Dis Child. 2015;100(6):565.

25. Ballard M, Schwarz R. Employing practitioner expertise in optimizing community healthcare systems. Healthcare J Delivery Sci Innov. 2018;S2213-0764(18)30022-8.

26. Bloom DE, Khoury A, Subbaraman R. The promise and peril of universal health care. Science. 2018;361(6404):eaat9644.

27. Scott K, Beckham S, Gross M, Pariyo G, Rao KD, Cometto G, et al. What do we know about community-based health worker programs? A systematic review of existing reviews on community health workers. Hum Resour Health. 2018;16(1):39.

28. He J, Irazola V, Mills KT, Poggio R, Beratarrechea A, Dolan J, et al. Effect of a community health worker-led multicomponent intervention on blood pressure control in low-income patients in Argentina: a randomized clinical trial. JAMA. 2017;318(11):1016-25

29. Sankaran S, Ravi PS, Wu YE, Shanabogue S, Ashok S, Agnew K, et al. An NGO-implemented community-clinic health worker approach to providing long-term care for hypertension in a remote region of southern India. Glob Health Sci Pract. 2017:5(4):668.

30. Nyarko KM, Ameme DK, Ocansey D, Commeh E, Markwei MT, Ohene SA Capacity assessment of selected health care facilities for the pilot implementation of Package for Essential Non-communicable Diseases (PEN) intervention in Ghana. Pan Afr Med J. 2016:25(Suppl 1):16.

31. World Health Organization. Implementation tools: Package of Essential Noncommunicable (PEN) disease interventions for primary health care in low-resource settings. Luxembourg: World Health Organization; 2013.

32. HEARTS. Technical package for cardiovascular disease management in primary health care. Geneva: WHO Press; 2018.

33. Wangchuk D, Virdi NK, Garg R, Mendis S, Nair N, Wangchuk D, et al. Package of essential noncommunicable disease (PEN) interventions in primary health-care settings of Bhutan: a performance assessment study. WHO South East Asia J Public Health. 2014;3(2):154-60.

34. Manjomo RC, Mwagomba B, Ade S, Ali E, Ben-Smith A, Khomani P, et al. Managing and monitoring chronic non-communicable diseases in a primary health care clinic, Lilongwe, Malawi. Public Health Action. 2016;6(2):60-5

35. Hyon CS, Nam KY, Sun HC, Garg R, Shrestha SM, Ok KU, et al. Package of Essential Noncommunicable Disease (PEN) interventions in primary healthcare settings in the Democratic People's Republic of Korea: a feasibility study. WHO South East Asia J Public Health. 2017;6(2):69-73.

36. Martinez RE, Quintana R, Go JJ, Villones MS, Marquez MA. Use of the WHO Package of Essential Noncommunicable Disease interventions after Typhoon Haiyan. Western Pac Surveill Response J. 2015;6(Suppl 1):18-20.

37. Kawamoto K, Houlihan CA, Balas EA, Lobach DF. Improving clinical practice using clinical decision support systems: a systematic review of trials to identify features critical to success. BMJ. 2005;330(7494):765
38. Algaze CA, Wood M, Pageler NM, Sharek PJ, Longhurst CA, Shin AY. Use of a checklist and clinical decision support tool reduces laboratory use and improves cost. Pediatrics. 2015;137(1):1-7.

39. O'Connor PJ, Desai JR, Butler JC, Kharbanda EO, Sperl-Hillen JM. Current status and future prospects for electronic point-of-care clinical decision support in diabetes care. Curr Diab Rep. 2013;13(2):172-6.

40. Nader CM, Tsevat J, Justice AC, Mrus JM, Levin F, Kozal MJ, et al. Development of an electronic medical record-based clinical decision support tool to improve HIV symptom management. AIDS Patient Care STDs. 2009;23(7):521-9.

41. Peiris DP, Joshi R, Webster RJ, Groenestein P, Usherwood TP, Heeley E, et al. An electronic clinical decision support tool to assist primary care providers in cardiovascular disease risk management: development and mixed methods evaluation. J Med Internet Res. 2009;11(4):e51.

42. Ayash CR, Simon SR, Marshall R, Kasper J, Chomitz V, Hacker K, et al. Evaluating the impact of point-of-care decision support tools in improving diagnosis of obese children in primary care. Obesity (Silver Spring). 2013;21(3):576-82.

43. Knoble SJ, Bhusal MR. Electronic diagnostic algorithms to assist mid-level health care workers in Nepal: a mixed-method exploratory study. Int J Med Inform. 2015;84(5):334-40.

44. Kallander K, Tibenderana JK, Akpogheneta OJ, Strachan DL, Hill Z, ten Asbroek $\mathrm{AH}$, et al. Mobile health (mHealth) approaches and lessons for increased performance and retention of community health workers in lowand middle-income countries: a review. J Med Internet Res. 2013;15(1):e17.

45. Clark J. Medicalization of global health 3: the medicalization of the noncommunicable diseases agenda. Glob Health Action. 2014;7(1):24002.

46. Khatib O. Noncommunicable diseases: risk factors and regional strategies for prevention and care. East Mediterr Health J. 2004;10(6):778-88.

47. Cost-effective strategies for noncommunicable diseases, risk factors, and behaviors. In: Jamison DT, Breman JG, Measham AR, Alleyne G, Claeson M, Evans DB, Jha P, Mills A, Musgrove P, editors. Priorities in health. Washington, DC: The International Bank for Reconstruction and Development/The World Bank; 2006: 97-128.

48. World Health Organization. Package of Essential Noncommunicable (PEN) disease and healthy lifestyle interventions: training modules for primary health care workers. New Delhi: World Health Organization ROfS-EA; 2018.

49. Health Services Administration (US). Chapter 3-motivational interviewing as a counseling style. In: Enhancing motivation for change in substance abuse treatment. Rockville: Substance Abuse and Mental Health Services Administration (US); 1999.

50. Rubak S, Sandbæk A, Lauritzen T, Christensen B. Motivational interviewing: a systematic review and meta-analysis. Br J Gen Pract. 2005;55(513):305-12.

51. Aira T, Wang W, Riedel M, Witte SS. Reducing risk behaviors linked to noncommunicable diseases in Mongolia: a randomized controlled trial. Am J Public Health. 2013;103(9):1666-74.

52. Heckman CJ, Egleston BL, Hofmann MT. Efficacy of motivational interviewing for smoking cessation: a systematic review and meta-analysis. Tob Control. 2010;19(5):410-6.

53. Ma C, Zhou Y, Zhou W, Huang C. Evaluation of the effect of motivational interviewing counselling on hypertension care. Patient Educ Couns. 2014; 95(2):231-7.

54. Ren $Y$, Yang H, Browning C, Thomas S, Liu M. Therapeutic effects of motivational interviewing on blood pressure control: a meta-analysis of randomized controlled trials. Int J Cardiol. 2014;172(2):509-11.

55. Channon S, Smith V, Gregory JW. A pilot study of motivational interviewing in adolescents with diabetes. Arch Dis Child. 2003;88(8):680-3.

56. World Health Organization. Disease and injury country estimates. https:// www.who.int/healthinfo/global_burden_disease/estimates_country/en/. Accessed 01 Feb 2019.

57. Aryal KK, Mehata S, Neupane S, Vaidya A, Dhimal M, Dhakal P, et al. The burden and determinants of non communicable diseases risk factors in Nepal: findings from a nationwide STEPS survey. PLoS One. 2015;10(8):e0134834.

58. Koirala B, Upreti S, Karmacharya B, Adhikari SR, Aryal KK, Kalaunee SP, Shrestha A, Vaidya A, Shrestha A, Jha AK, Shrestha B, Karki KB, Neupane D, Pande VK, Karki A, Koju R, Ojha SP, Pant BR, Raut A, Sharma SK, Sharma D, Dhimal M, Mishra SR, Oli N, Pandey AR, Kalaunee A, Mehata S, Paudel P, Bhatia A, Coates M, Bukhman G, Gupta N, Schwarz D. Report. Nepal NCDI Poverty Commission (group authorship). The Nepal NCDI Poverty Commission: An equity initiative to address non-communicable diseases and injuries. National Report 2018. Kathmandu: Nepal NCDI Poverty Commission. 2018.

59. Upreti SR, Lohani GR, Magtymova A, Dixit LP. Strengthening policy and governance to address the growing burden of diabetes in Nepal. WHO South East Asia J Public Health. 2016;5(1):40-3. 
60. Constitution of Nepal, 2015 (2072). Edited by Nepal Go. Kathmandu: Government of Nepal; 2015. p. 175

61. Ministry of Health and Population, Government of Nepal. Multisectoral Action Plan for the Prevention and Control of Non Communicable Diseases (2014-2020). Kathmandu: Government of Nepal. 2014.

62. Mishra SR, Kallestrup P, Neupane D. Country in focus: confronting the challenge of NCDs in Nepal. Lancet Diabetes Endocrinol. 2016;4(12):979-80.

63. Schwarz D, Sharma R, Bashyal C, Schwarz R, Baruwal A, Karelas G, et al. Strengthening Nepal's Female Community Health Volunteer network: a qualitative study of experiences at two years. BMC Health Serv Res. 2014;14:473.

64. Glenton C, Scheel IB, Pradhan S, Lewin S, Hodgins S, Shrestha V. The female community health volunteer programme in Nepal: decision makers' perceptions of volunteerism, payment and other incentives. Soc Sci Med. 2010;70(12):1920-7.

65. Maes KC, Kohrt BA, Closser S. Culture, status and context in community health worker pay: pitfalls and opportunities for policy research. A commentary on Glenton et al. (2010). Soc Sci Med. 2010;71(8):1375-8.

66. Neupane D, McLachlan CS, Mishra SR, Olsen MH, Perry HB, Karki A, et al. Effectiveness of a lifestyle intervention led by female community health volunteers versus usual care in blood pressure reduction (COBIN): an openlabel, cluster-randomised trial. Lancet Global Health. 2018:6(1):e66-73.

67. Knoble S, Pandit A, Koirala B, Ghimire L. Measuring the quality of ruralbased, government health care workers in Nepal. Internet J Allied Health Sci Pract. 2010;8(1):1-36.

68. Curran GM, Bauer M, Mittman B, Pyne JM, Stetler C. Effectivenessimplementation hybrid designs: combining elements of clinical effectiveness and implementation research to enhance public health impact. Med Care. 2012;50(3):217-26.

69. Bernet AC, Willens DE, Bauer MS. Effectiveness-implementation hybrid designs: implications for quality improvement science. Implement Sci. 2013;8(1):S2.

70. Poudel KC, Okumura J, Sherchand JB, Jimba M, Murakami I, Wakai S. Mumbai disease in far western Nepal: HIV infection and syphilis among male migrantreturnees and non-migrants. Tropical Med Int Health. 2003;8(10):933-9.

71. Vaidya NK, Wu J. HIV epidemic in far-western Nepal: effect of seasonal labor migration to India. BMC Public Health. 2011;11(1):310.

72. Seddon D, Adhikari J, Gurung G. Foreign labor migration and the remittance economy of Nepal. Crit Asian Stud. 2002;34(1):19-40.

73. Thapa D, Ghimire I. Labour migration for employment: a status report for Nepal: 2014/2015. Edited by Ministry of Labour and Employment. Kathmandu: Government of Nepal; 2016.

74. Field Bulletin: why do people migrate? An overview of labour migration issues in Achham and Bajura Kathmandu, Nepal. Kathmandu: Ministry of Health and Population, Government of Nepal; 2013.

75. Ministry of Health and Population, New ERA, ICF International Inc. Nepal Demographic and Health Survey 2016. Kathmandu: Ministry of Health and Population; 2017.

76. United Nations Development Programme. Government of Nepal National Planning Commission. Nepal Human Development Report 2014: beyond geography—unlocking human potential. Kathmandu: United Nations Development Programme, Government of Nepal National Planning Commission; 2014

77. Government of Nepal, National Planning Commission. Nepal Earthquake 2015: post disaster needs assessment. Kathmandu: Government of Nepal, National Planning Commission 2015.

78. Citrin D, Thapa P, Nirola I, Pandey S, Kunwar LB, Tenpa J, et al. Developing and deploying a community healthcare worker-driven, digitally-enabled integrated care system for municipalities in rural Nepal. Healthcare J Delivery Sci Innov. 2018;6(3):197-204.

79. Palinkas LA, Aarons GA, Horwitz S, Chamberlain P, Hurlburt M, Landsverk J. Mixed method designs in implementation research. Admin Pol Ment Health. 2011;38(1):44-53.

80. Glasgow RE, Vogt TM, Boles SM. Evaluating the public health impact of health promotion interventions: the RE-AIM framework. Am J Public Health. 1999;89(9):1322-7.

81. Chan A, Tetzlaff JM, Altman DG, et al. SPIRIT 2013 Statement: Defining Standard Protocol Items for Clinical Trials. Ann Intern Med. 2013;158:200-207.

82. American Diabetes Association. 6. Glycemic targets: standards of medical care in diabetes-2018. Diabetes Care. 2018;41(Supplement 1):S55-64

83. Arnett DK, Blumenthal RS, Albert MA, Michos ED, Buroker AB, Miedema MD, et al. ACC/AHA Guideline on the Primary Prevention of Cardiovascular Disease. J Am Coll Cardiol. 2019;2019:26029.
84. Vogelmeier CF, Criner GJ, Martinez FJ, Anzueto A, Barnes PJ, Bourbeau J, et al. Global strategy for the diagnosis, management, and prevention of chronic obstructive lung disease 2017 report. GOLD executive summary. Am J Respir Crit Care Med. 2017;195(5):557-82.

85. Özaltın A, Cashin C. Costing of health services for provider payment: a practical manual based on country costing challenges, trade-offs, and solutions. Washington, DC: Joint Learning Network for Universal Health Coverage; 2014

86. Schwarz D, Schwarz R, Gauchan B, Andrews J, Sharma R, Karelas G, et al. Implementing a systems-oriented morbidity and mortality conference in remote rural Nepal for quality improvement. BMJ Qual Saf. 2011;20(12): 1082-8.

87. Harris PA, Taylor R, Thielke R, Payne J, Gonzalez N, Conde JG. Research electronic data capture (REDCap) — a metadata-driven methodology and workflow process for providing translational research informatics support. J Biomed Inform. 2009;42(2):377-81.

88. Strauss A, Corbin JM. Basics of qualitative research: grounded theory procedures and techniques. London: Sage; 1990.

89. Bernard HR. Handbook of methods in cultural anthropology. Walnut Creek: Altamira Press; 1998.

90. QIP Ltd. NVivo qualitative data analysis software. Version 10 edn. Burlington: QSR International; 2012.

\section{Publisher's Note}

Springer Nature remains neutral with regard to jurisdictional claims in published maps and institutional affiliations.

\section{Ready to submit your research? Choose BMC and benefit from:}

- fast, convenient online submission

- thorough peer review by experienced researchers in your field

- rapid publication on acceptance

- support for research data, including large and complex data types

- gold Open Access which fosters wider collaboration and increased citations

- maximum visibility for your research: over $100 \mathrm{M}$ website views per year

At BMC, research is always in progress.

Learn more biomedcentral.com/submissions 